OPEN ACCESS

Edited by:

Ana Maria Otero,

University of Santiago

de Compostela, Spain

Reviewed by:

Giordano Rampioni,

Roma Tre University, Italy

Wim J. Quax,

University of Groningen, Netherlands

*Correspondence: Rodolfo García-Contreras

rgarc@bq.unam.mx

Specialty section:

This article was submitted to Microbial Physiology and Metabolism,

a section of the journal

Frontiers in Microbiology

Received: 15 May 2019

Accepted: 31 October 2019

Published: 14 November 2019

Citation:

López-Jácome LE

Garza-Ramos G,

Hernández-Durán $M$,

Franco-Cendejas $R$, Loarca $D$

Romero-Martínez D, Nguyen PTD,

Maeda T, González-Pedrajo B,

Díaz-Guerrero M, Sánchez-Reyes JL,

Díaz-Ramírez $D$ and

García-Contreras R (2019) AiiM

Lactonase Strongly Reduces Quorum Sensing Controlled Virulence Factors in Clinical Strains of Pseudomonas aeruginosa Isolated From Burned

Patients. Front. Microbiol. 10:2657.

doi: 10.3389/fmicb.2019.02657

\section{AiiM Lactonase Strongly Reduces Quorum Sensing Controlled Virulence Factors in Clinical Strains of Pseudomonas aeruginosa Isolated From Burned Patients}

\author{
Luis Esaú López-Jácome1,2, Georgina Garza-Ramos³, Melissa Hernández-Durán², \\ Rafael Franco-Cendejas ${ }^{2}$, Daniel Loarca', Daniel Romero-Martínez ${ }^{3}$, \\ Phuong Thi Dong Nguyen ${ }^{4}$, Toshinari Maeda ${ }^{4}$, Bertha González-Pedrajo ${ }^{5}$, \\ Miguel Díaz-Guerrero ${ }^{5}$, Jorge Luis Sánchez-Reyes ${ }^{1}$, Dánae Díaz-Ramírez ${ }^{1}$ and \\ Rodolfo García-Contreras ${ }^{1 *}$
}

\footnotetext{
' Laboratorio de Bacteriología, Departamento de Microbiología y Parasitología, Facultad de Medicina, Universidad Nacional Autónoma de México, Mexico City, Mexico, ${ }^{2}$ Laboratorio de Infectología, Centro Nacional de Investigación y Atención de Quemados, Instituto Nacional de Rehabilitación, Mexico City, Mexico, ${ }^{3}$ Laboratorio de Fisicoquímica e Ingeniería de Proteínas, Departamento de Bioquímica, Universidad Nacional Autónoma de México, Mexico City, Mexico, ${ }^{4}$ Department of Biological Functions Engineering, Gradute School of Life Sciences and System Engineering, Kyushu Institute of Technology, Kitakyushu, Japan, ${ }^{5}$ Departamento de Genética Molecular, Instituto de Fisiología Celular, Universidad Nacional Autónoma de México, Mexico City, Mexico
}

Pseudomonas aeruginosa is an opportunistic bacterium associated with healthcare infections in intensive care units (ICUs), ventilator-associated pneumonia (VAP), surgical site infections, and burns. This bacterium causes $75 \%$ of death in burned patients, since it can develop a persistent biofilm associated with infections, express several virulence factors, and antibiotic-resistance mechanisms. Some of these virulence factors are proteases such as elastase and alkaline protease, or toxic metabolites such as pyocyanin and is one of the few microorganisms able to produce cyanide, which inhibits the cytochrome oxidase of host cells. These virulence factors are controlled by quorum sensing (QS). In this work, $30 P$. aeruginosa clinical strains isolated from burned patients from a tertiary hospital in Mexico City were studied. Antibiotic susceptibility tests were done, and virulence factors (elastase, alkaline protease, $\mathrm{HCN}$, and pyocyanin) were determined in presence of an $\mathrm{N}$-acylhomoserine lactonase, AiiM able to hydrolyze a wide range of acyl homoserine lactones. The treatment reduced significantly the activities of elastase and alkaline protease, and the production of pyocyanin and HCN in all producer strains but not the secretion of toxins through the type III secretion system. Our work suggests that AiiM treatment may be an effective therapy to combat $P$. aeruginosa infection in burn patients.

Keywords: AiiM lactonase, virulence factors, Pseudomonas aeruginosa, quorum quenching, burned patients, anti-virulence therapy 


\section{INTRODUCTION}

Pseudomonas aeruginosa is an opportunistic bacteria associated with healthcare infections in intensive care units (ICUs), ventilator-associated pneumonia (VAP), central line-associated blood stream infections, surgical site infections (Cohen et al., 2017), burnt wounds (Fournier et al., 2016), and urinary tract infections, otitis media, and keratitis (Chatterjee et al., 2016; Olivares et al., 2016). In the United States, according to the Centers for Disease Control and Prevention, in 2013 it was estimated that every year around 51,000 health-care infections are associated to $P$. aeruginosa, of which 6,700 are multidrug resistant, causing 440 deaths per year (Centers for Disease Control and Prevention, 2018) ${ }^{1}$. Both P. aeruginosa and Acinetobacter baumannii complex are the most important, resistant and dangerous microorganisms infecting burnt patients (Tredget et al., 1992; Estahbanati et al., 2002; Turner et al., 2014; Centers for Disease Control and Prevention, 2019). Despite medicine advances, these sorts of complications are still a huge problem to solve, and as a consequence, around $75 \%$ of burned infected patients die. Burn infections related to $P$. aeruginosa often promote a faster deterioration allowing the spread of bacteria causing death in weeks and even in days (Mcmanus et al., 1985; Turner et al., 2014). P. aeruginosa has a wide arsenal of virulence factors that enable it to colonize and cause infections in the host, the relevance of these virulence factors has been demonstrated using $P$. aeruginosa strains with deficiencies in their production, leading to a reduced ability of colonizing and a lower dissemination in the host (Pavlovskis and Wretlind, 1979; Rumbaugh et al., 2009; Jimenez et al., 2012; CastilloJuarez et al., 2015). Elastase is a metalloprotease that disrupts several proteins such as: collagen, elastin, immunoglobulins (IgA and IgG), complement components, and cytokines like interferon gamma and tumor necrosis factor alpha (Pavlovskis and Wretlind, 1979; Lyczak et al., 2000; Ben Haj Khalifa et al., 2011). Alkaline protease is also a zinc metalloprotease that inhibits phagocytosis, killing through neutrophils, opsonization, the action of the complement cascade by degrading C3b and is as well related to corneal damage (Howe and Iglewski, 1984; Ben Haj Khalifa et al., 2011; Laarman et al., 2012; Lee and Zhang, 2015). P. aeruginosa is one of the few microorganisms that can synthesize cyanide through the oxidative decarboxylation of glycine by hydrogen cyanide synthase enzyme, under microaerobic conditions $\left(\mathrm{O}_{2}<5 \%\right)$. $\mathrm{HCN}$ is a poison that inhibits respiration by inactivating cytochrome oxidase $\mathrm{C}$ (Huber et al., 2016). Another important virulence factor is pyocyanin, a blue phenazine, that promotes oxidative stress, which inhibits ciliary movement and delays inflammatory response due to the damage of neutrophils and apoptosis induction (Ben Haj Khalifa et al., 2011; Lee and Zhang, 2015). In burn injuries, pyocyanin plays an important role because it stimulates colonization, damage of surrounding tissue and promotes dissemination. Furthermore, $P$. aeruginosa strains are often multi-drug resistant, limiting treatment options in healthcare settings around the globe, owing this the World Health Organization classified P. aeruginosa as

${ }^{1}$ https://www.cdc.gov/hai/organisms/pseudomonas.html the second more threatening bacterium. Moreover, although new antibiotics are available, each time, resistance against those new drugs quickly appears (Fournier et al., 2016; Shortridge et al., 2017; Karampatakis et al., 2018; Shields et al., 2018). In many pathogenic bacteria, virulence factors are controlled by cell to cell communication known as quorum sensing (QS). P. aeruginosa has two QS systems mediated by $\mathrm{N}$-acyl homoserine lactones, Las and $\mathrm{Rhl}$, each one is constituted by three elements, a synthase, a signal receptor and an autoinducer signal. The Las system is formed by LasI which is the synthase, the receptor is LasR and the autoinducer is N-3-oxo-dodecanoyl-L-homoserine lactone, meanwhile the Rhl system is formed by the synthase RhlI, RhlR as receptor and the auto-inducer is N-butyryl-homoserine lactone. These two systems are hierarchically organized and each one of them controls several virulence factors. The Las system regulates the Rhl system and virulence factors such as elastase, protease, exotoxin A, alkaline protease and type II secretion system; while the Rhl system enhances the production of rhamnolipids, hydrogen cyanide, and pyocyanin (Van Delden and Iglewski, 1998; Douzi et al., 2011; Lee and Zhang, 2015). Due to the fast increase in bacterial resistance, alternative strategies such as quorum quenching (QQ) have been proposed. QQ consists of blocking or inhibiting cell to cell communication by obstructing the autoinducer synthases, the signal receptors or by degrading the autoinducers via two enzymatic strategies: disrupting the lactone ring through a lactonase or through the cleavage of the acylic tail by acylases (Defoirdt et al., 2013; Defoirdt, 2018). The aim of this work is to evaluate the activity of AiiM, a lactonase enzyme, in $P$. aeruginosa clinical bacterial strains isolated from burnt patients in a third level center of Mexico City, in order to find out if its utilization could eventually be proposed to treat infected burnt patients.

\section{MATERIALS AND METHODS}

\section{Clinical Strains}

Randomly 200 strains were selected $^{2}$ from the collection belonging to Infectious Diseases Laboratory at Centro Nacional de Investigación y Atención de Quemados at Instituto Nacional de Rehabilitación Luis Guillermo Ibarra, to avoid genomic redundancy pulsed field, gel electrophoresis was performed and only one strain per clonal group was selected for further experiments. Clinical strains were identified with Vitek 2 Compact $^{\circledR}$ (Biomerieux, France) with Gram negative card identification, some biochemical tests included were oxidase, indole production, growth at $42^{\circ} \mathrm{C}$, arginine dihydrolase and glucose oxidation/fermentation. The origin of each clinical isolate is shown in Supplementary Table S1.

\section{Minimal Inhibitory Concentrations}

Minimal inhibitory concentrations were determined according to Clinical and Laboratory Standards Institute ${ }^{\circledR}$ (CLSI) M07A10 (CLSI, 2015) in 96-well plates. Breakpoints interpretation were made according to the M100 Performance Standards for

\footnotetext{
${ }^{2}$ www.randomization.com
} 
Antimicrobial susceptibility testing 28th edition (CLSI, 2019). Antibiotics included were amikacin (Sigma Aldrich A1774), gentamicin (Sigma Aldrich G3632), aztreonam (Sigma Aldrich PZ0038), ceftazidime (Sigma Aldrich C3809), cefepime (Sigma Aldrich PHR1763), ciprofloxacin (Sigma Aldrich 17850), levofloxacin (Sigma Aldrich 28266), doripenem (Sigma Aldrich 32138), imipenem (Sigma Aldrich I0160), meropenem (Sigma Aldrich M2574), colistin (Sigma Aldrich C4461), and piperacillin/tazobactam (Sigma Aldrich P8396/T2820). P. aeruginosa ATCC ${ }^{\circledR} 27853$ was used as control as according to CLSI (Supplementary Table S1).

\section{las/rhl Genes Detection DNA Extraction}

Pseudomonas aeruginosa strains were cultured in 5\% sheep blood agar during $18 \mathrm{~h}$ at $37^{\circ} \mathrm{C}$, and then one single colony was taken and lysed in an Eppendorf tube with $500 \mu \mathrm{L}$ of TE buffer $(10 \mathrm{mM}$ Tris-HCl, 1 mM EDTA, pH 7.5) and were set into a heat block at $95^{\circ} \mathrm{C}$ for $5 \mathrm{~min}$. Tubes were centrifuged, and the supernatant was added into a new tube.

Genes related to Las and $\mathrm{Rhl}$ systems were amplified (Table 1) in a final volume of $25 \mu \mathrm{L}$ of buffer $1 \mathrm{X}, 3 \mathrm{mM}$ $\mathrm{MgCl}_{2}, 200 \mu \mathrm{M} d \mathrm{dNTP}^{\prime} \mathrm{s}, 0.2 \mu \mathrm{M}$ primer forward and reverse, $0.026 \mathrm{U} / \mu \mathrm{L}$ Taq polymerase (Amplitaq Gold ${ }^{\circledR}$ DNA Polymerase, Applied Biosystems N808-0241, United States). The amplification conditions used were: $95^{\circ} \mathrm{C} 10 \mathrm{~min}, 95^{\circ} \mathrm{C} 30 \mathrm{~s}$, $58^{\circ} \mathrm{C} 45 \mathrm{~s}$ and $72^{\circ} \mathrm{C} 50 \mathrm{~s}$ during 35 cycles, $72^{\circ} \mathrm{C} 5 \mathrm{~min}$ and finally $4^{\circ} \mathrm{C}$ (Veriti 96 Well thermal cycler, Applied Biosystems, United States). Amplification products were loaded into a $1 \%$ agarose gel stained with SYBR ${ }^{\circledR}$ green I (S7567, Life Technologies, United States) and visualized with Gel DOC ${ }^{\mathrm{TM}} \mathrm{XR}+$ with Image $\mathrm{Lab}^{\mathrm{TM}}$ software (Bio-Rad, United States). P. aeruginosa PAO1 was used as positive control in each one of the systems and $\Delta$ $l a s R / r h l R$ PAO1 as negative control of transcriptional regulators.

\section{AiiM Purification}

AiiM construction was provided by Dr. Toshinari Maeda (Nguyen et al., 2019). Briefly $50 \mathrm{~mL}$ Escherichia coli M15/pQE30 AiiM was grown overnight $(\mathrm{ON})$ in Luria Bertani broth with $100 \mu \mathrm{g} / \mathrm{mL}$ of carbenicillin (Sigma Aldrich C1389) and $50 \mu \mathrm{g} / \mathrm{mL}$ of kanamycin (Sigma Aldrich K1876), afterward, $10 \mathrm{~mL}$ of the ON cultures were taken and inoculated into $1 \mathrm{~L}$ of terrific broth with carbenicillin and kanamycin as above described, cultures were incubated at $37^{\circ} \mathrm{C} 220 \mathrm{rpm}$, optical density (OD)

TABLE 1 | Primers used in this study.

\begin{tabular}{|c|c|c|}
\hline Primer & $\operatorname{Tm}\left({ }^{\circ} \mathrm{C}\right)$ & Size (bp) \\
\hline lasl-F 5'-CGCGAAGAGTTCGATAAA-3' & 59.7 & 531 \\
\hline lasl-R 5'-GGTCTTGGCATTGAGTTC-3' & 58.7 & \\
\hline lasR-F 5'-ATGGCCTTGGTTGACGGT-3’ & 65.9 & 706 \\
\hline lasR-R 5'-GACCCAAATTAACGGCCA-3' & 63.7 & \\
\hline rhll-F 5'-TTGCTCTCTGAATCGCTG-3' & 61 & 590 \\
\hline$r h l l-R$ 5'-GCCATCGACAGCGGTACG-3' & 68.3 & \\
\hline$r h / R-F$ 5'-ATGAGGAATGACGGAGGC-3' & 63.2 & 675 \\
\hline rhIR-R 5'-CGCGTCGAACTTCTTCTG-3' & 62.9 & \\
\hline
\end{tabular}

at $600 \mathrm{~nm}$ was measured until the culture reached an OD of 0.5 and immediately after, it was induced with $500 \mu \mathrm{M}$ IPTG. The cultures were incubated at $37^{\circ} \mathrm{C} 220 \mathrm{rpm}$ for $6 \mathrm{~h}$ and centrifuged at 10,000 rpm for $40 \mathrm{~min}$. Pellets were resuspended in $40 \mathrm{~mL}$ of purification buffer $\left(50 \mathrm{mM} \mathrm{NaH} \mathrm{PO}_{4}, 300 \mathrm{mM}\right.$ $\mathrm{NaCl}$ and $10 \%$ glycerol, $\mathrm{pH}$ adjusted to 8.0 ) and $500 \mu \mathrm{M}$ PMSF (Sigma Aldrich P7626), sonicated (45\% amplitude for $45 \mathrm{~s}$ and 2 min of rest, all this 10 times; Ultrasonic processor, Cole Parmer) and centrifuged at 10,000 rpm during $40 \mathrm{~min}$. The supernatants were passed through a $0.2 \mathrm{~nm}$ filter and loaded onto Protino ${ }^{\circledR}$ Ni-TED resin (Macherey-Nagel, 745200.600) for purification of His-tagged proteins previously equilibrated with 3 column volumes (CV) of $20 \mathrm{~mL}$ of purification buffer, then filtered protein extracts were passed through the column, after that, 2 additional CV of purification buffer were passed. Protein was eluted with $150 \mathrm{mM}$ imidazole (Sigma Aldrich I5513) in $2 \mathrm{CV}$ of purification buffer. AiiM fractions with higher purity were selected, concentrated with polyethylene glycol $35 \mathrm{KDa}$ (Sigma Aldrich 946-46) into dialysis tubing cellulose membrane (Sigma Aldrich D9777). Afterward, dialysis was done to remove imidazole using dialysis buffer $(50 \mathrm{mM}$ Tris, $300 \mathrm{mM} \mathrm{NaCl}$ adjusted at $\mathrm{pH} 7.5)$. SDS-PAGE was done to estimate the amount and purity of AiiM. Protein was quantified by its absorbance at $280 \mathrm{~nm}$ with NanoDrop 2000 (Thermo Fisher Scientific, United States), using an extinction coefficient (Abs $0.1 \%$ ) of 1.08. Aliquots of protein were made and stored at $-20^{\circ} \mathrm{C}$ until they were used.

\section{AiiM Activity Against $\mathrm{N}$-acyl Homoserine Lactones}

To evaluate the HSL lytic activity of the purified AiiM, an analytical assay was developed in an Alliance HPLC system (Waters, United States) with a Symmetry (Waters, United States) C18 Column (75 mm, $3.5 \mathrm{~mm})$. Both short and long acylated chains were included, $1 \mathrm{mM} N$-butyryl-DLhomoserine lactone (C4-HSL; Sigma Aldrich 09945), 1 mM N-(3oxooctanoyl)-L-homoserine lactone (3OC8-HSL;Sigma Aldrich O1764), $1 \mathrm{mM} N$-decanoyl-DL-homoserine lactone (C10HSL; Sigma Aldrich 17248), 1 mM N-(3-oxodecanoyl)-Lhomoserine lactone (3OC10-HSL; Sigma Aldrich O9014), and $\mathrm{N}$-(3-oxododecanoyl) homoserine lactone (3OC12-HSL; Sigma Aldrich O9139). $60 \mathrm{mM} \mathrm{NaOH}$ was used as positive control since it can hydrolyze HSL molecules and the reaction was stopped with the addition of $2 \mathrm{~N} \mathrm{HCl}$. Several concentrations, from 250, 100, 50, 25, 10, and $5 \mu \mathrm{g} / \mathrm{mL}$ of purified AiiM were tested. Time exposition was also varied; $24 \mathrm{~h}, 2 \mathrm{~h}$, $1 \mathrm{~h}, 30 \mathrm{~min}, 20 \mathrm{~min}, 10 \mathrm{~min}$, and $5 \mathrm{~min}$. All experiments were performed in triplicate. Chromatographic conditions were: column temperature $25^{\circ} \mathrm{C}$, sample temperature $25^{\circ} \mathrm{C}$, injection volume $10 \mu \mathrm{L}$, flow rate $1 \mathrm{~mL} / \mathrm{min}$, detection $205 \mathrm{~nm}$. Elution mixture was made with $50 \mathrm{mM}$ phosphates buffer $\mathrm{pH}$ 2.9:acetonitrile. For C4-HSL the relation was 90:10, 3OC8-HSL 60:40, C10-HSL 60:40, 3OC10-HSL 60:40, and 3OC12-HSL 50:50.

\section{Bacterial Growth With and Without AiiM}

Grow curves were analyzed to test the effect of AiiM on $P$. aeruginosa PAO1 and $\Delta$ lasR/rhlR PAO1 growth kinetics. 
A $50 \mathrm{~mL}$ flask with $5 \mathrm{~mL}$ of $\mathrm{LB}$ was inoculated with each one of the strains at an initial $\mathrm{OD}_{600 \mathrm{~nm}}$ of 0.05 with and without $5 \mu \mathrm{g} / \mathrm{mL}$ of AiiM, samples were taken each hour for $12 \mathrm{~h}$.

\section{Virulence Factors Determination}

Elastase was determined for clinical strains and $P$. aeruginosa PAO1 $\Delta l a s R / r h l R$ and $\Delta l a s I / r h l I$ PAO1 mutants according to methods previously described (Ohman et al., 1980), with some modifications. $\mathrm{ON}$ of each clinical strain and control strains were cultured in $\mathrm{LB}$ at $37^{\circ} \mathrm{C} 220 \mathrm{rpm}$ and were inoculated at an initial $\mathrm{OD}_{600 \mathrm{~nm}}$ of 0.05 in $5 \mathrm{~mL}$ of $\mathrm{LB}$ with and without AiiM $(5 \mu \mathrm{g} / \mathrm{mL})$, samples were incubated for $18 \mathrm{~h}$ at $37^{\circ} \mathrm{C}$ and $220 \mathrm{rpm}$, and centrifuged at $14,000 \mathrm{rpm}$ for $2 \mathrm{~min}$. $50 \mu \mathrm{L}$ of the supernatant were taken and set into $950 \mu \mathrm{L}$ of elastase buffer (100 mM Tris- $\left.\mathrm{HCl}, 1 \mathrm{mM} \mathrm{CaCl}_{2}, \mathrm{pH} 7.5\right)$ with $2.5 \mathrm{mg}$ of elastin-congo red (Sigma Aldrich reference E0502) as substrate. Tubes were incubated at $37^{\circ} \mathrm{C}, 220 \mathrm{rpm}$ for $2 \mathrm{~h}$, centrifuged at 14,000 rpm for $5 \mathrm{~min}$ and the released die in the supernatant was measured at $495 \mathrm{~nm}$ with a spectrophotometer SmartSpec Plus (Bio-Rad, United States). All determinations were performed by triplicate.

\section{Alkaline Protease}

Alkaline protease was determined according to methods previously described (Howe and Iglewski, 1984), with some modifications. $\mathrm{ON}$ of each strains were cultured into $\mathrm{LB}$ at $37^{\circ} \mathrm{C}$ $220 \mathrm{rpm}$ and were inoculated in $5 \mathrm{~mL}$ of $\mathrm{LB}$ at an initial $\mathrm{OD}_{600 \mathrm{~nm}}$ of 0.05 with and without AiiM $(5 \mu \mathrm{g} / \mathrm{mL})$. AiiM protein was added at the beginning of the cultures, samples were incubated for $18 \mathrm{~h}$ at $37^{\circ} \mathrm{C}$ and $220 \mathrm{rpm}$, and centrifuged at $14,000 \mathrm{rpm}$ for $2 \mathrm{~min}$. $50 \mu \mathrm{L}$ of supernatant were taken and added into $950 \mu \mathrm{L}$ of protease buffer with $2.5 \mathrm{mg}$ of Hide-Remazol brilliant blue R (Sigma Aldrich reference H6268) as substrate. Tubes were incubated at $37^{\circ} \mathrm{C}, 220 \mathrm{rpm}$ for $20 \mathrm{~min}$ centrifuged at $14,000 \mathrm{rpm}$ $5 \mathrm{~min}$ and the supernatant was measured at $595 \mathrm{~nm}$ with a spectrophotometer SmartSpec Plus (Bio-Rad, United States).

\section{Pyocyanin}

For pyocyanin production $\mathrm{ON}$ of each clinical and control strains were cultured in $\mathrm{LB}$ at $37^{\circ} \mathrm{C}$ at $220 \mathrm{rpm}$ and inoculated into $5 \mathrm{~mL}$ of $\mathrm{LB}$ at an initial $\mathrm{OD}_{600 \mathrm{~nm}} 0.05$ with and without AiiM $(5 \mu \mathrm{g} / \mathrm{mL})$. AiiM protein was added at the beginning, samples were incubated $18 \mathrm{~h}$ at $37^{\circ} \mathrm{C}$ and $220 \mathrm{rpm}$. One milliliter of supernatant was taken, centrifuged at $14,000 \mathrm{rpm}$ for $5 \mathrm{~min}$, and then $800 \mu \mathrm{L}$ of supernatant were set into new $1.5 \mathrm{~mL}$ conic tube and $400 \mu \mathrm{L}$ of chloroform was added, each tube was mixed in vortex for $2 \mathrm{~min}$. Tubes were centrifuged for $5 \mathrm{~min}$ at $14,000 \mathrm{rpm}$, $300 \mu \mathrm{L}$ of the organic phase were taken and deposited into a new tube, $800 \mu \mathrm{L}$ of $0.2 \mathrm{~N} \mathrm{HCl}$ were added and mixed for $2 \mathrm{~min}$ in vortex then samples were read at $520 \mathrm{~nm}$ (Maeda et al., 2012). P. aeruginosa PAO1, $\Delta$ lasR/rhlR PAO1 and $\Delta$ lasI/rhlI PAO1 were used as positive and negative controls, respectively.

\section{HCN Determination}

For HCN determination, bacteria were cultured in $3 \mathrm{~mL}$ of LB medium in flasks with rubber stoppers at $37^{\circ} \mathrm{C}$ and $200 \mathrm{rpm}$ for $18 \mathrm{~h}$, after the incubation two needles were inserted in the rubber stopper, one of them was used for pumping air for $1 \mathrm{~h}$ and the other to collect the outflow in $5 \mathrm{~mL}$ of 4 $\mathrm{M} \mathrm{NaOH}$. HCN concentrations were determined as described by Gallagher and Manoil (2001), briefly, samples were mixed with a 1:1 fresh mixture of $0.1 \mathrm{M} o$-dinitrobenzene and 0.2 M p-nitrobenzaldehyde both dissolved in 2-methoxyethanol, and following $20 \mathrm{~min}$ of incubation at room temperature, the absorbance at $578 \mathrm{~nm}$ was determined and compared with a calibration curve made with KCN standards.

\section{AiiM Dose Response Curve and Suppression of Its Activity by Exogenous Addition of 30C12-HSL}

For these control experiments, the PAO1 reference strain and the clinical isolate P809 were used. Three independent cultures per strain were inoculated in LB medium at an initial $\mathrm{OD}_{600 \mathrm{~nm}}$ of 0.05 without and with AiiM at $0.5,1,2.5$, and $5 \mu \mathrm{g} / \mathrm{mL}$, and incubated $37^{\circ} \mathrm{C}$ at $220 \mathrm{rpm}$, supernatants were then obtained and used for the determination of pyocyanin concentration and elastase activity (as described before). In addition another 3 cultures per strain with AiiM $0.5 \mu \mathrm{g} / \mathrm{mL}$ were grown to an $\mathrm{OD}_{600 \mathrm{~nm}}$ of $\sim 1.0$, supplemented with a final concentration of $30 \mu \mathrm{M}$ of $3 \mathrm{OC} 12$ HSL, and incubated until $18 \mathrm{~h}$ of incubation were completed, supernatants were collected and pyocyanin concentration and elastase activity determined.

\section{Long Chain HSL Autoinducer Detection and Its Inactivation by AiiM}

To identify autoinducer production and its inactivation by AiiM, each one of the 30 clinical strains were grew up onto MacConkey agar plates, then one colony was taken and inoculated into $5 \mathrm{~mL}$ of LB for ON growth. After that, new cultures were inoculated at an initial $\mathrm{OD}_{600 \mathrm{~nm}} 0.05$ in $5 \mathrm{~mL}$ of $\mathrm{LB}$ and grown at $37^{\circ} \mathrm{C}$, with $220 \mathrm{rpm}$ shaking during $18 \mathrm{~h}$, with AiiM $5 \mu \mathrm{g} / \mathrm{mL}$ and without AiiM enzyme. LB cultures then were centrifuged 14,000 rpm for $5 \mathrm{~min}$. Supernatants were separated in new tubes. For long chain HSL detection Agrobacterium tumefaciens NT1 pZLR4 (Shaw et al., 1997) was used as a biosensor strain. Previously the biosensor strain was grown in one liter of M9 medium and incubated at $37^{\circ} \mathrm{C}$ and $220 \mathrm{rpm}$ for $18 \mathrm{~h}$. Bacteria were concentrated by centrifugation at $12,000 \mathrm{rpm}$ during $5 \mathrm{~min}$ to a final volume of $15 \mathrm{~mL}$, then aliquots of $1 \mathrm{~mL}$ of concentrated bacteria were separated. M9 agar plates were prepared and before solidification $1 \mathrm{~mL}$ of the concentrated biosensor plus Xgal at a final concentration of $40 \mu \mathrm{g} / \mathrm{mL}$ (5-bromo-4-chloro3-indolyl- 3 -D-galactopyranoside, USB corporation, Cleveland, $\mathrm{OH}$, United States) were added for $100 \mathrm{~mL}$ of M9 agar. $15 \mu \mathrm{L}$ of each supernatant (with and without AiiM) were then added onto $6 \mathrm{~mm}$ filter paper sterile disk on the M9 agar. All experiments were done by triplicate. $1 \mathrm{mM} \mathrm{N}$-decanoyl-DLhomoserine lactone (C10-HSL; Sigma Aldrich 17248), $1 \mathrm{mM}$ $\mathrm{N}$-(3-oxodecanoyl)-L-homoserine lactone (3OC10-HSL; Sigma Aldrich O9014) and $N$-(3-oxododecanoyl) homoserine lactone (3OC12-HSL; Sigma Aldrich O9139) were used as positive controls and the molecules treated with AiiM $5 \mu \mathrm{g} / \mathrm{mL}$ as negative controls. Plates were incubated at $28^{\circ} \mathrm{C}$ and results were observed, a positive reaction associated to the production of long chain HSL was observed as a green halo and inactivation of the signals when the halo was absent. 


\section{Type III Protein Secretion Profiles}

For type III secretion assays $P$. aeruginosa strains (PAO1, PA14, and the clinical isolates H015 and P729) were grown overnight in LB medium. Bacteria were diluted 1:200 into $4 \mathrm{~mL}$ of a modified LB medium supplemented with $10 \mathrm{mM}$ $\mathrm{MgCl}_{2}, 0.5 \mathrm{mM} \mathrm{CaCl}$ and $5 \mathrm{mM}$ EGTA (pH 7.4) in the presence or absence of $5 \mu \mathrm{g} / \mathrm{mL}$ of AiiM, and grown at $37^{\circ} \mathrm{C}$ to an $\mathrm{OD}_{600 \mathrm{~nm}}$ of 0.8 to $1.0 .1 \mathrm{~mL}$ of each culture was collected into a microcentrifuge tube and bacteria were pelleted by centrifugation. The resulting pellet was resuspended in $200 \mu \mathrm{l}$ of $1 \times$ Laemmli SDS sample buffer normalized for $\mathrm{OD}_{600 \mathrm{~nm}}$. The supernatant was centrifuged once again and the resulting supernatant was transferred into a clean tube. Supernatant proteins were precipitated overnight at $4^{\circ} \mathrm{C}$ by adding trichloroacetic acid to a final concentration of $10 \%$, pelleted by centrifugation and resuspended in $20 \mu \mathrm{L}$ of $1 \mathrm{x}$ Laemmli SDS sample buffer containing 10\% saturated Tris base normalized for $\mathrm{OD}_{600 \mathrm{~nm}}$. Samples were separated by $15 \%$ SDS-PAGE, transferred onto a nitrocellulose membrane and probed for the presence of the effectors ExoS and ExoU by immunoblotting. Detection was performed using the Immobilon Western chemiluminescent HRP substrate (Millipore), and bands were visualized on X-ray films (Carestream MXB Film).

\section{B-Lactams Inactivation}

As B-lactams have a lactone ring, we performed the inactivation disk method to determine whether AiiM would be able to inactivate this kind of antibiotics. Briefly, $2 \mathrm{~mL}$ of $0.8 \%$ isotonic saline solution (ISS) was added into sterile $12 \mathrm{~mm} \times 75 \mathrm{~mm}$ tube, disks of $30 \mu \mathrm{g}$ ceftazidime (Becton Dickinson, United States), $30 \mu \mathrm{g}$ cefepime (Becton Dickinson, United States), $10 \mu \mathrm{g}$ imipenem (Becton Dickinson, United States), and $10 \mu \mathrm{g}$ meropenem (Becton Dickinson, United States). One set of disks containing ISS was used as negative control, another set of all antibiotics above mentioned with $5 \mu \mathrm{g} / \mathrm{mL}$ of AiiM, and finally, since $\mathrm{NaOH}$ can break the lactone ring another set of all antibiotics was used as positive control with $60 \mathrm{mM} \mathrm{NaOH}$. Escherichia coli ATCC ${ }^{\circledR} 25922$ was used as a pansusceptible strain. Disks were incubated for 1 and $10 \mathrm{~min}, 2$ and $24 \mathrm{~h}$. A suspension of 0.5 McFarland was made with E. coli ATCC ${ }^{\circledR} 25922$ and was plated onto Müller Hinton agar (Becton Dickinson, United States), tubes were incubated at $37^{\circ} \mathrm{C}$ until their use. All experiments were made by triplicate; the inhibition diameter was measured using a Vernier device.

\section{RESULTS}

All clinical isolates were obtained from burned patients infected with $P$. aeruginosa. The most common burn etiology was fire (66.7\%) followed by scalds (23.3\%) and electrical burns (10\%). Medians of hospital length of stay were 53 days (8-303), the mean total body surface area was $40 \%(10-85 \%)$ with a mortality rate of $26.6 \%(n=8)$. $P$. aeruginosa strains were isolated from urine $(n=8)$, quantitative biopsies $(n=8)$, blood $(n=6)$, endotracheal aspirates $(n=3)$, bronchoalveolar lavage fluid $(n=2)$, catheter tips $(n=2)$, and qualitative biopsy $(n=1)$.

\section{Pseudomonas aeruginosa Antibiotic Susceptibility Patterns}

Susceptibility tests were carried out for all clinical isolates with different antibiotic families including aminoglycosides, monobactams, cephalosporins, fluoroquinolones, carbapenems, lipopeptides, and B-lactam combination agents. The strains were resistant to almost all antibiotics except colistin (Figure 1), resistance rates against all antibiotics families were over $50 \%$. The highest resistance rates were for carbapenems which, until recent decades, were the most potent antibiotics against $P$. aeruginosa and other non-fermentative Gram negative rods; therefore colistin represents the last treatment option for these types of infections (Supplementary Table S1).

\section{Gene Amplification}

In order to determine if $\mathrm{Las} / \mathrm{Rhl}$ systems were present in P. aeruginosa isolated from burned patients, PCR was performed using the primers described in Table 1. Results showed that the genes encoding the Las system (lasI and lasR) and Rhl system ( $r h l I$ and $r h l R$ ) were found in all the clinical strains. P. aeruginosa PAO1 was used as positive control and $\triangle l a s R / r h l R$ PAO1 was used as negative control (data not shown).

\section{AiiM Purification}

AiiM protein was obtained at a purity of $>90 \%$ as judged by SDS-PAGE. After elution with $150 \mathrm{mM}$ imidazole, fractions were collected, and those in which AiiM was present were concentrated into dialysis tubing cellulose membrane with polyethylene glycol, then protein concentration was determined and kept at $-20^{\circ} \mathrm{C}$ until used. The purified protein consists of a single band of $\approx$ $27 \mathrm{kDa}$, compared with a theoretical molecular mass of $27.2 \mathrm{kDa}$ (Supplementary Figure S1).

\section{AiiM Activity Against Homoserine Lactones}

In order to prove AiiM activity against acylated chains of diverse HSL, cleavage was determined by HPLC. Five HSL autoinducers with both short and long chains were tested (C4HSL, 3OC8-HSL, C10-HSL, 3OC10-HSL, and 3OC12-HSL). First HSL alone was run to identify retention times (Supplementary Table S2), later the same HSL were treated with $60 \mathrm{mM} \mathrm{NaOH}$ to disrupt the lactone ring and finally HSL molecules were treated with AiiM and HPLC experiments were performed under the same conditions. Several AiiM concentrations $(250 \mu \mathrm{g} / \mathrm{mL}$, $100 \mu \mathrm{g} / \mathrm{mL}, 50 \mu \mathrm{g} / \mathrm{mL}, 25 \mu \mathrm{g} / \mathrm{mL}, 10 \mu \mathrm{g} / \mathrm{mL}$, and $5 \mu \mathrm{g} / \mathrm{mL}$ ) were used, in order to identify the lowest one suitable for inhibiting the expression of virulence factors. Each HSL was used at $1 \mathrm{mM}$. The lowest concentration of AiiM tested $(5 \mu \mathrm{g} / \mathrm{mL})$ was enough to cleave all the HSLs in 5 min (Figure 2 and Supplementary Figure S2) and therefore, this concentration was used for all subsequent experiments.

\section{AiiM Does Not Affect Pseudomonas aeruginosa Growth}

Growth curves of P. aeruginosa with and without AiiM were done to determine if its addition had any effect in the growth rates. 


\section{Clinical $P$. aeruginosa susceptibility patterns}

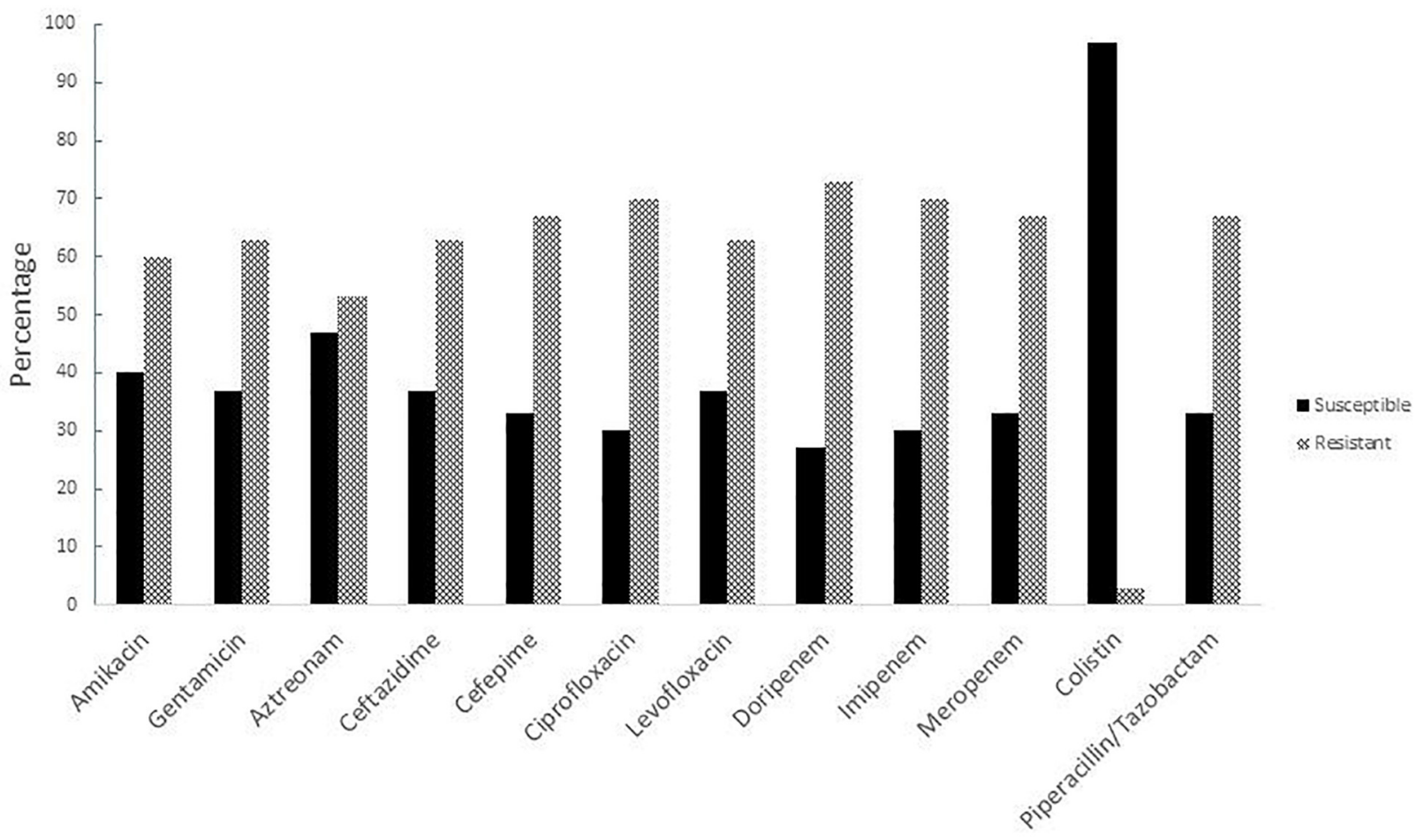

FIGURE 1 | Susceptibility patterns of clinical $P$. aeruginosa strains isolated from burned patients.

As expected, there was no difference in the growing dynamics between these cultures (Supplementary Figure S3). PAO1 and $\Delta l a s R / r h l R$ strains were used as controls.

\section{QS-Controlled Virulence Factors Inhibition}

Once it was confirmed that AiiM did not affect $P$. aeruginosa growth, its effect over the expression of the QS-controlled virulence factors was determined. Experiments were classified in two groups, one without AiiM addition, and the other with $5 \mu \mathrm{g} / \mathrm{mL}$ addition of AiiM. For elastolytic activity (Figure 3A), activity was found in 29 clinical samples, while for alkaline protease activity (Figure 3B) there were 27 producing strains; only 12 strains produced pyocyanin (Figure 3C), and seven strains were $\mathrm{HCN}$ producers (Figure $3 \mathrm{D}$ ). At the same time, experiments with AiiM addition were carried out and the same virulence factors were measured. A significant decrease in the production of each virulence factor was found (elastase $p=0.000002$, protease $p=0.000004$, pyocyanin $p=0.001$ and $p=0.008$ for $\mathrm{HCN})$.

In addition, AiiM dose response experiments using it at $0.5,1$, 2.5 and $5 \mu \mathrm{g} / \mathrm{mL}$ were performed with the reference strain PAO1 and the clinical isolate P809 measuring pyocyanin production and elastase activity, as expected the degree of inhibition of both phenotypes was dependent in the concentration of AiiM for both virulence factors (Supplementary Figure S4). Moreover the effect of $0.5 \mu \mathrm{g} / \mathrm{mL}$ was partially reversed by the addition of $30 \mu \mathrm{M}$ of 3OC12-HSL (Supplementary Figure S4). In order to verify that the clinical strains had active QS systems and that the inhibitory effect in the expression of QS-dependent virulence factors exerted by AiiM was mediated by the degradation of QS signals. Identification of long chain HSL for each strain was done using the biosensor strain Agrobacterium tumefaciens NT1 pZLR4 (Shaw et al., 1997), as expected all strains were long chain HSL producers, moreover also for all strains AiiM at $5 \mu \mathrm{g} / \mathrm{mL}$ was enough to degrade the long chain HSL of all strains as determined with the biosensor strain (Supplementary Figure S5).

\section{AiiM Does Not Inhibit the Type III Secretion System}

Despite its strong inhibitory activity against QS-controlled virulence factors, AiiM had no effect on the secretion of T3SS effectors in both PA14 and PAO1 type strains as well as in the clinical strains P729 and $\mathrm{H} 015$ at $5 \mu \mathrm{g} / \mathrm{mL}$ (Supplementary Figure S6) and even using $20 \mu \mathrm{g} / \mathrm{mL}$ (data not shown). These two clinical strains were selected as representative examples of a secretion profile similar to strains PAO1 or PA14, respectively.

\section{AiiM Does Not Inactivate B-Lactam Antibiotics}

Wang et al. (2010) defined AiiM as a member of the superfamily of alpha/beta hydrolases, this may represent a problem if it has the ability to inactivate the broad spectrum of $B$-lactam antibiotics 

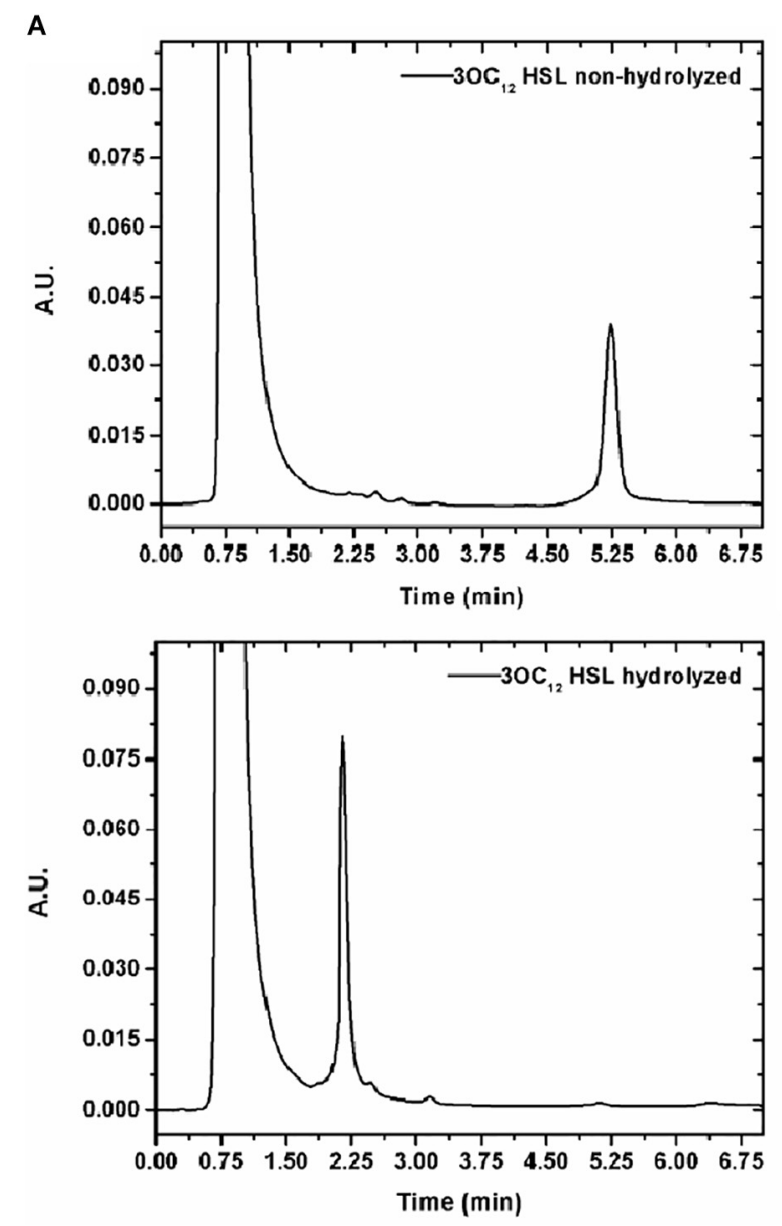
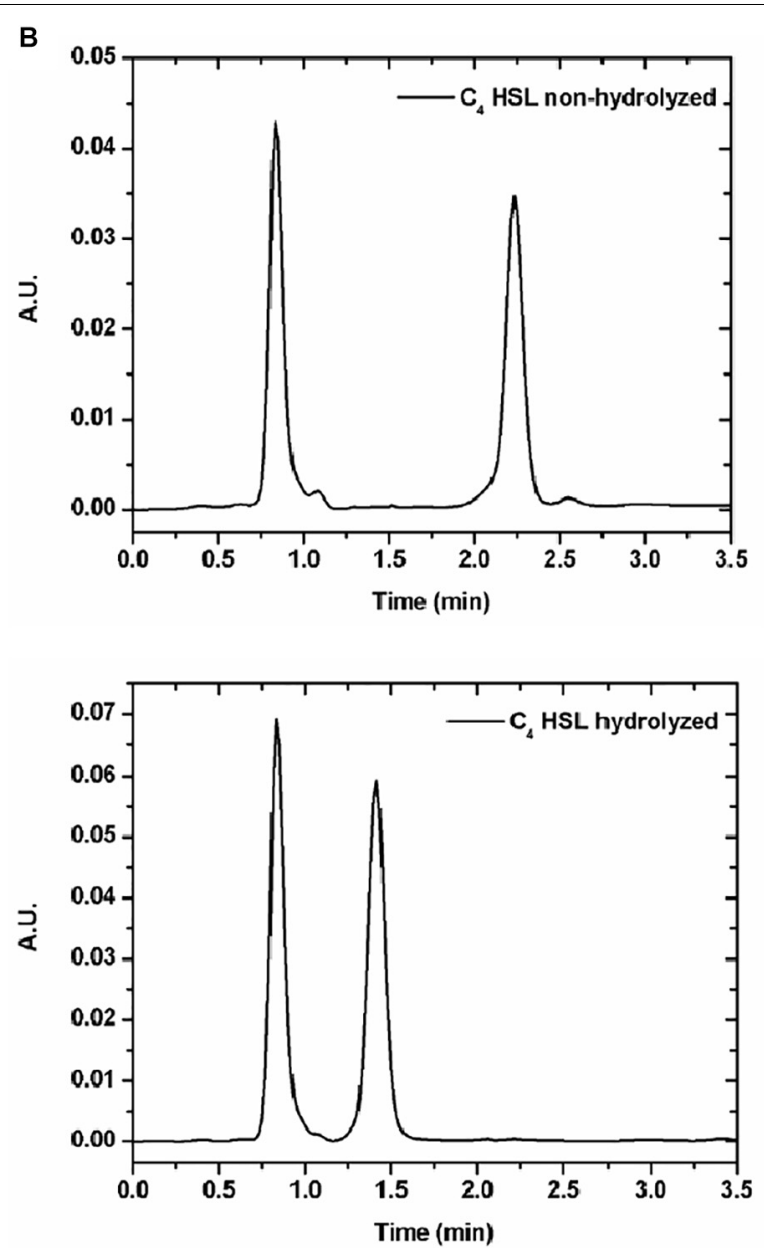

FIGURE 2 | (A) HPLC of HSL without (above) and with $5 \mu \mathrm{g} / \mathrm{mL}$ of AiiM (below) after 5 min of exposition. 2A N-(3-oxododecanoyl) homoserine lactone. Retention time for non-hydrolyzed HSL is 5.3 min whereas for hydrolyzed is 2.24 min. (B) 2B N-butyryl-DL-homoserine lactone. Retention time for non-hydrolyzed is 2.29 min whereas for hydrolyzed is $1.42 \mathrm{~min}$.

as other carbapenem enzymes do, such as NDM, IMP or VIM. In order to test this, we exposed anti Pseudomonas ß-lactam antibiotics to $5 \mu \mathrm{g} / \mathrm{mL}$ of AiiM (Supplementary Figures S7, S8). Nevertheless, AiiM did not degrade any anti Pseudomonas B-lactam antibiotics.

\section{DISCUSSION}

Pseudomonas aeruginosa is one of the main bacteria that causes hospital acquired infections in immunocompromised patients and vulnerable ones (Azam and Khan, 2018). $P$. aeruginosa is one of the 12 priority multi-drug resistant bacteria according to the WHO list published in 2017 (WHO, 2017) and belongs to the ESKAPE group, together with Enterococcus faecium, Staphylococcus aureus, Klebsiella pneumoniae, A. baumannii, and Enterobacter species (Chen et al., 2018). In addition to acquired resistance mechanisms such as carbapenemases (Carmeli et al., 2016), P. aeruginosa has many intrinsic antibiotic tolerance mechanisms, for instance low permeability in its external membrane, and expression of several efflux pumps (Malhotra et al., 2018; Ferrer-Espada et al., 2019).

Burn injuries are one of the most common and devastating forms of trauma and patients with serious thermal injury require immediate specialized care in order to minimize morbidity and mortality (Church et al., 2006). P. aeruginosa is one of the most frequent bacteria associated to infection in burn patients together with A. baumannii (Li et al., 2018). In a recent Mexican study (Garza-Gonzalez et al., 2019), P. aeruginosa had around $27 \%$ of resistance to carbapenems, in a global context it was one of the main bacteria in 47 Mexican health centers in 20 states, 175/1995 strains were multi-drug-resistant, 165/1995 were possible extreme drug resistant and 87/1995 possible pandrug resistant. In our 30 isolates we had more than $60 \%$ of resistance to cephalosporins (ceftazidime and cefepime), carbapenems (dorypenem, imipenem, and meropenem), aminoglycosides (amikacin and gentamicin), fluoroquinolones (ciprofloxacin and levofloxacin), and piperacillin/tazobactam. Moreover, one strain was resistant to colistin, which is the last antibiotic resource. 


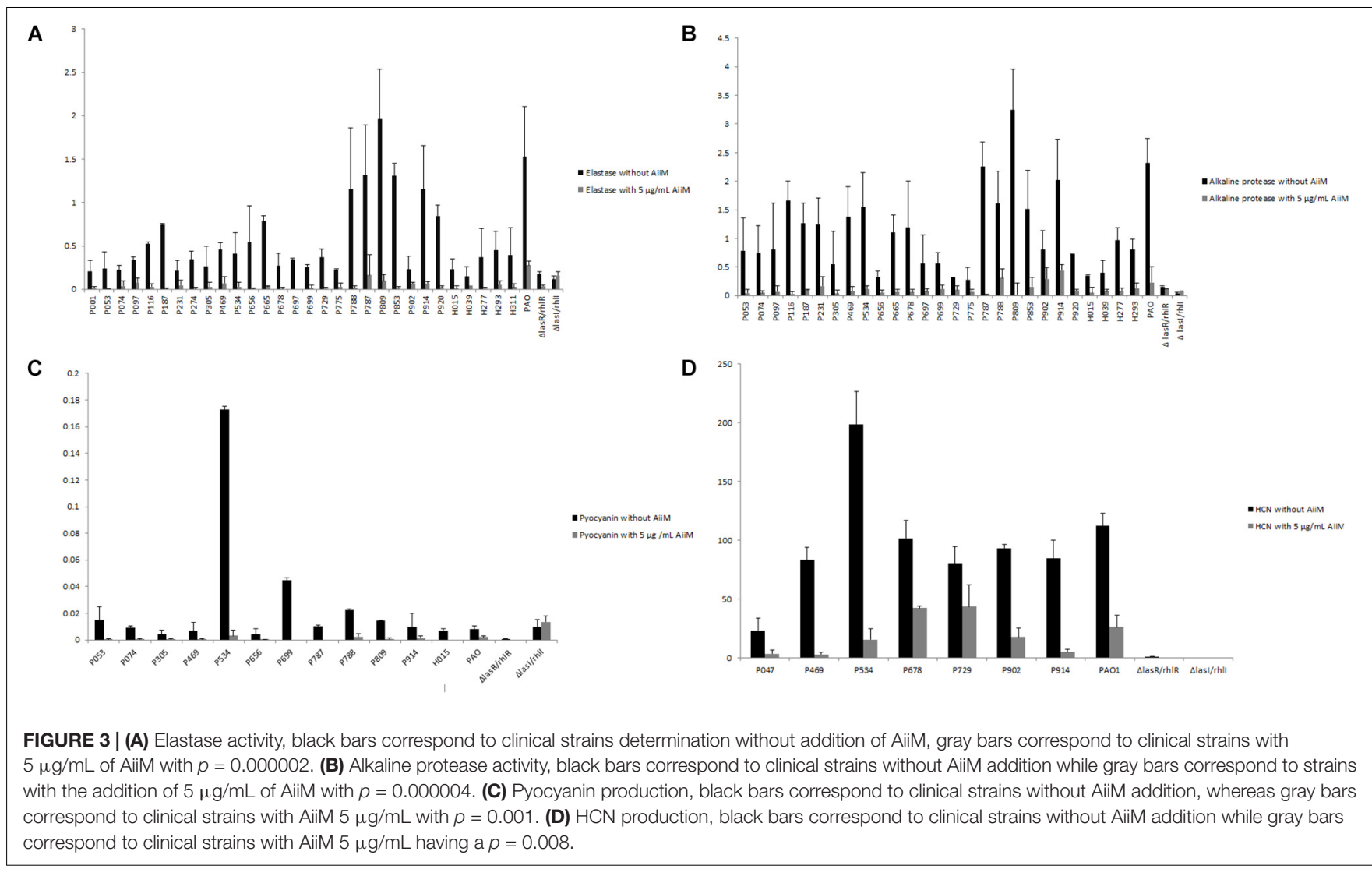

AiiM showed a wide activity and was able to cut all HSL molecules tested, consistent with a previous report by Wang et al. (2010). Even though we did not analyze it in a quantitative form, we infer a strong activity of AiiM due to its ability to degrade all HSL tested within $5 \mathrm{~min}$ of exposition, moreover in our study $5 \mu \mathrm{g} / \mathrm{mL}$ were enough to break down these molecules. One of the main characteristics that a quorum quencher must fulfill is that it should not inhibit bacterial growth (Defoirdt et al., 2013; Defoirdt, 2018) and as expected, AiiM treatment did not affect $P$. aeruginosa growth kinetic.

In contrast, AiiM significantly reduced the four QS-dependent virulence factors tested in our study following a dose response pattern; moreover, others have demonstrated that AiiM had very good activity in a mouse model of acute pneumonia (Migiyama et al., 2013) and reduces methane production in waste sewage sludge (Nguyen et al., 2018). Although to date, the majority of studies with QQ enzymes have been performed only in type strains like PAO1, PA14 (Fetzner, 2015), recently, Guendouze et al. (2017) did the first investigation with $P$. aeruginosa clinical strains isolated from diabetic foot using the lactonase SsoPox with a substitution in the amino acid 263 changing a tryptophan to isoleucine, in order to increase the enzymatic activity, using $0.5 \mathrm{mg} / \mathrm{mL}$ of protein, they found some strains with certain tolerance to the SsoPox addition. In our study, AiiM reduced elastase and alkaline protease activities, pyocyanin and HCN concentrations, and we did not find any strain with tolerance against AiiM treatment, in spite that AiiM was used at a 100 times lower concentration than SsoPox. Moreover, AiiM effectivity is much higher than the effectivity of small molecule QS inhibitors such as brominated furanones and 5-fluorouracil, that cannot inhibit QS-virulence factor production of several of the clinical strains tested, and that are very toxic to some of them (GarcíaContreras et al., 2013, 2015; García-Contreras, 2016; GarcíaContreras et al., 2016; Guendouze et al., 2017).

Nevertheless, for type III secretion, no inhibition by AiiM was found, which is consistent with recent findings showing that in a $\Delta l a s R / r h l R$ mutant of $P$. aeruginosa PAO1, T3SS effector toxins are secreted at the same levels than in the wild-type strain, demonstrating that this virulence factor is not positively regulated by QS (Soto-Aceves et al., 2019), instead it may be used at low cell densities to establish infections in the host (Hauser, 2009). These highlights the importance of targeting both QS and T3SS to develop robust anti-virulence therapies (García-Contreras, 2016). Moreover, other results indicate that the inhibition of QS systems and T3SS by molecules such as coumarin (Zhang et al., 2018) must be due to independent effects over the QS systems and T3SS.

One possible limitation of the utilization of AiiM and other QQ enzymes for treating $P$. aeruginosa infections is the fact that lasR defective mutants are often found in infections and although in principle these mutants will produce low levels of QS-dependent virulence factors, this is not always the case due to a rewiring of the virulence factor regulation 
(Morales et al., 2017). And these strains could be tolerant against the effect of QQ enzymes.

Since AiiM is a member of the alpha/beta hydrolases superfamily and several antibiotics are inactivated by metallo $ß$ lactamases, reducing clinical options to treat infections (Hong et al., 2015), we tested if AiiM could cleave these ß-lactamase antibiotics, however, AiiM did not inactivate those tested, and hence it could be safely used in combination with them.

Although in vivo tests in burn infection models are lacking, our work suggests that AiiM treatment may be an effective addition for the treatment of $P$. aeruginosa infections, and since research by other groups had shown also the utility of the lactonase SsoPox against clinical isolates from diabetic foot patients in vitro (Guendouze et al., 2017) and in vivo using an amoeba model (Mion et al., 2019), and in rat pneumonia against the PAO1 strain (Hraiech et al., 2014) lactonase utilization became an strong candidate for its eventual application in the clinical practice, moreover although in vivo studies using acylases are scarce, recently it was shown the PvdQ in addition to their inhibitory properties in vitro (Sio et al., 2006) and in Caenorhabditis elegans model was also able to increase survival, reduce damage and decrease bacterial loads in a pulmonary infection mice model (Papaioannou et al., 2009; Utari et al., 2018). Hence QQ enzymes may be beneficial for the treatment of burn and lung infections as well.

\section{CONCLUSION}

AiiM showed a strong activity against C4-HSL, 3OC8HSL, C10-HSL, 3OC10-HSL, and 3OC12-HSL. It reduced elastase and alkaline protease activities as well as pyocyanin and HCN concentrations in all tested clinical strains of $P$. aeruginosa isolated from burned patients and no AiiM tolerant strain was found. However, it had no inhibitory effect against the T3SS.

\section{DATA AVAILABILITY STATEMENT}

All datasets generated for this study are included in the article/Supplementary Material.

\section{REFERENCES}

Azam, M. W., and Khan, A. U. (2018). Updates on the pathogenicity status of Pseudomonas aeruginosa. Drug. Discov. Today 24, 350-359. doi: 10.1016/j. drudis.2018.07.003

Ben Haj Khalifa, A., Moissenet, D., Vu Thien, H., and Khedher, M. (2011). Virulence factors in Pseudomonas aeruginosa: mechanisms and modes of regulation. Ann. Biol. Clin. 69, 393-403. doi: 10.1684/abc.2011.0589

Carmeli, Y., Armstrong, J., Laud, P. J., Newell, P., Stone, G., Wardman, A., et al. (2016). Ceftazidime-avibactam or best available therapy in patients with ceftazidime-resistant Enterobacteriaceae and Pseudomonas aeruginosa complicated urinary tract infections or complicated intra-abdominal infections (REPRISE): a randomised, pathogen-directed, phase 3 study. Lancet Infect. Dis. 16, 661-673. doi: 10.1016/S1473-3099(16)30004-4

\section{ETHICS STATEMENT}

Pseudomonas aeruginosa clinical strains used in this study were isolated as part of routine clinical hospital procedures to diagnose infection and hence ethical approval was not required, according to the National Institute of Rehabilitation ethical committee. All bacterial isolates were stored as part of laboratory and epidemiology necessities.

\section{AUTHOR CONTRIBUTIONS}

LL-J, GG-R, MH-D, DR-M, PN, MD-G, DL, JS-R, and DD-R performed the experiments. RF-C, TM, BG-P, GG-R, LL-J, and $\mathrm{RG}-\mathrm{C}$ designed the study, supervised the project and discussed the results. LL-J wrote the manuscript with input from all authors.

\section{FUNDING}

This work was supported by Grants from the Programa de Apoyo a Proyectos de Investigación e Innovación Tecnológica (PAPIIT), the Universidad Nacional Autónoma de México number IN214218, the Consejo Nacional de Ciencia y Tecnología number SEP-CONACYT CB-A1-S-8530, and the CONACYT INFR-2015-252140 grant.

\section{ACKNOWLEDGMENTS}

RG-C is grateful to Beatriz Meráz Rios for her assistance with some experiments. We acknowledge Dr. Norma Espinosa Sánchez and Eugenia Flores Robles for technical assistance. LL-J is a doctoral student from the Programa de Doctorado en Ciencias Biomédicas, Universidad Nacional Autónoma de México.

\section{SUPPLEMENTARY MATERIAL}

The Supplementary Material for this article can be found online at: https://www.frontiersin.org/articles/10.3389/fmicb. 2019.02657/full\#supplementary-material

Castillo-Juarez, I., Maeda, T., Mandujano-Tinoco, E. A., Tomas, M., Perez-Eretza, B., Garcia-Contreras, S. J., et al. (2015). Role of quorum sensing in bacterial infections. World J Clin Cases 3, 575-598. doi: 10.12998/wjcc.v3.i7.575

Centers for Disease Control and Prevention, (2018). Pseudomonas aeruginosa in Healthcare Settings. Available at: https://www.cdc.gov/hai/organisms/ pseudomonas.html (accessed March 9, 2018).

Centers for Disease Control and Prevention, (2019). Diseases and Organisms in Healthcare Settings. Atlanta GA: Centers for Disease Control and Prevention.

Chatterjee, M., Anju, C. P., Biswas, L., Anil Kumar, V., Gopi Mohan, C., and Biswas, R. (2016). Antibiotic resistance in Pseudomonas aeruginosa and alternative therapeutic options. Int. J. Med. Microbiol. 306, 48-58. doi: 10.1016/j.ijmm. 2015.11.004

Chen, J. W., Lau, Y. Y., Krishnan, T., Chan, K. G., and Chang, C. Y. (2018). Recent advances in molecular diagnosis of Pseudomonas aeruginosa infection 
by state-of-the-art genotyping techniques. Front. Microbiol 9:1104. doi: 10. 3389/fmicb.2018.01104

Church, D., Elsayed, S., Reid, O., Winston, B., and Lindsay, R. (2006). Burn wound infections. Clin. Microbiol. Rev 19, 403-434.

CLSI, (2015). M07-A10 Methods for Dilution Antimicrobial Susceptibility Test for Bacteria that Grow Aerobically; Approved Standard, 10th Edn. Wayne, PA: Clinical and Laboratory Standards Institute.

CLSI, (2019). M100 Performance Standards for Antimicrobial Susceptibility Testing, 29th Edn. Wayne, PA: Clinical and Laboratory Standards Institute.

Cohen, R., Babushkin, F., Cohen, S., Afraimov, M., Shapiro, M., Uda, M., et al. (2017). A prospective survey of Pseudomonas aeruginosa colonization and infection in the intensive care unit. Antimicrob. Resist. Infect. Control 6:7.

Defoirdt, T. (2018). Quorum-sensing systems as targets for antivirulence therapy. Trends Microbiol. 26, 313-328. doi: 10.1016/j.tim.2017.10.005

Defoirdt, T., Brackman, G., and Coenye, T. (2013). Quorum sensing inhibitors: how strong is the evidence? Trends Microbiol 21, 619-624. doi: 10.1016/j.tim. 2013.09.006

Douzi, B., Ball, G., Cambillau, C., Tegoni, M., and Voulhoux, R. (2011). Deciphering the Xcp Pseudomonas aeruginosa type II secretion machinery through multiple interactions with substrates. J. Biol. Chem. 286, 40792-40801. doi: 10.1074/jbc.M111.294843

Estahbanati, H. K., Kashani, P. P., and Ghanaatpisheh, F. (2002). Frequency of Pseudomonas aeruginosa serotypes in burn wound infections and their resistance to antibiotics. Burns 28, 340-348. doi: 10.1016/s0305-4179(02)00024-4

Ferrer-Espada, R., Shahrour, H., Pitts, B., Stewart, P. S., Sanchez-Gomez, S., and Martinez-De-Tejada, G. (2019). A permeability-increasing drug synergizes with bacterial efflux pump inhibitors and restores susceptibility to antibiotics in multi-drug resistant Pseudomonas aeruginosa strains. Sci. Rep. 9:3452. doi: 10.1038/s41598-019-39659-4

Fetzner, S. (2015). Quorum quenching enzymes. J. Biotechnol. 201, 2-14. doi: 10.1016/j.jbiotec.2014.09.001

Fournier, A., Voirol, P., Krahenbuhl, M., Bonnemain, C. L., Fournier, C., Pantet, O., et al. (2016). Antibiotic consumption to detect epidemics of Pseudomonas aeruginosa in a burn centre: a paradigm shift in the epidemiological surveillance of Pseudomonas aeruginosa nosocomial infections. Burns 42, 564-570. doi: 10.1016/j.burns.2015.10.030

Gallagher, L. A., and Manoil, C. (2001). Pseudomonas aeruginosa PAO1 kills Caenorhabditis elegans by cyanide poisoning. J. Bacteriol. 183, 6207-6214. doi: 10.1128/jb.183.21.6207-6214.2001

García-Contreras, R. (2016). Is quorum sensing interference a viable alternative to treat Pseudomonas aeruginosa infections? Front. Microbiol. 7:1454. doi: 10. 3389/fmicb.2016.01454

García-Contreras, R., Maeda, T., and Wood, T. K. (2016). Can resistance against quorum-sensing interference be selected? ISME J. 10, 4-10. doi: 10.1038/ismej. 2015.84

García-Contreras, R., Martinez-Vazquez, M., Velazquez Guadarrama, N., Villegas Paneda, A. G., Hashimoto, T., Maeda, T., et al. (2013). Resistance to the quorum-quenching compounds brominated furanone C-30 and 5-fluorouracil in Pseudomonas aeruginosa clinical isolates. Pathog. Dis. 68, 8-11. doi: 10.1111/ 2049-632X.12039

García-Contreras, R., Perez-Eretza, B., Jasso-Chavez, R., Lira-Silva, E., RoldanSanchez, J. A., Gonzalez-Valdez, A., et al. (2015). High variability in quorum quenching and growth inhibition by furanone C-30 in Pseudomonas aeruginosa clinical isolates from cystic fibrosis patients. Pathog. Dis. 73:ftv040. doi: 10.1093/ femspd/ftv040

Garza-Gonzalez, E., Morfin-Otero, R., Mendoza-Olazaran, S., Bocanegra-Ibarias, P., Flores-Trevino, S., Rodriguez-Noriega, E., et al. (2019). A snapshot of antimicrobial resistance in Mexico. Results from 47 centers from 20 states during a six-month period. PLoS One 14:e0209865. doi: 10.1371/journal.pone. 0209865

Guendouze, A., Plener, L., Bzdrenga, J., Jacquet, P., Remy, B., Elias, M., et al. (2017). Effect of quorum quenching lactonase in clinical isolates of Pseudomonas aeruginosa and comparison with quorum sensing inhibitors. Front. Microbiol. 8:227. doi: $10.3389 /$ fmicb. 2017.00227

Hauser, A. R. (2009). The type III secretion system of Pseudomonas aeruginosa: infection by injection. Nat. Rev. Microbiol. 7, 654-665. doi: 10.1038/ nrmicro2199
Hong, D. J., Bae, I. K., Jang, I. H., Jeong, S. H., Kang, H. K., and Lee, K. (2015). Epidemiology and characteristics of metallo-beta-lactamase-producing Pseudomonas aeruginosa. Infect. Chemother. 47, 81-97. doi: 10.3947/ic.2015.47. 2.81

Howe, T. R., and Iglewski, B. H. (1984). Isolation and characterization of alkaline protease-deficient mutants of Pseudomonas aeruginosa in vitro and in a mouse eye model. Infect. Immun. 43, 1058-1063.

Hraiech, S., Hiblot, J., Lafleur, J., Lepidi, H., Papazian, L., Rolain, J. M., et al. (2014). Inhaled lactonase reduces Pseudomonas aeruginosa quorum sensing and mortality in rat pneumonia. PLoS One 9:e107125. doi: 10.1371/journal.pone. 0107125

Huber, P., Basso, P., Reboud, E., and Attree, I. (2016). Pseudomonas aeruginosa renews its virulence factors. Environ. Microbiol. Rep. 8, 564-571. doi: 10.1111/ 1758-2229.12443

Jimenez, P. N., Koch, G., Thompson, J. A., Xavier, K. B., Cool, R. H., and Quax, W. J. (2012). The multiple signaling systems regulating virulence in Pseudomonas aeruginosa. Microbiol. Mol. Biol. Rev. 76, 46-65. doi: 10.1128/MMBR.05007-11

Karampatakis, T., Antachopoulos, C., Tsakris, A., and Roilides, E. (2018) Molecular epidemiology of carbapenem-resistant Pseudomonas aeruginosa in an endemic area: comparison with global data. Eur. J. Clin. Microbiol. Infect. Dis. 37, 1211-1220. doi: 10.1007/s10096-018-3244-4

Laarman, A. J., Bardoel, B. W., Ruyken, M., Fernie, J., Milder, F. J., Van Strijp, J. A., et al. (2012). Pseudomonas aeruginosa alkaline protease blocks complement activation via the classical and lectin pathways. J. Immunol. 188, 386-393. doi: 10.4049/jimmunol.1102162

Lee, J., and Zhang, L. (2015). The hierarchy quorum sensing network in Pseudomonas aeruginosa. Protein Cell 6, 26-41. doi: 10.1007/s13238-0140100-x

Li, L., Dai, J. X., Xu, L., Chen, Z. H., Li, X. Y., Liu, M., et al. (2018). Antimicrobial resistance and pathogen distribution in hospitalized burn patients: a multicenter study in Southeast China. Medicine 97:e11977. doi: 10.1097/MD.0000000000011977

Lyczak, J. B., Cannon, C. L., and Pier, G. B. (2000). Establishment of Pseudomonas aeruginosa infection: lessons from a versatile opportunist. Microbes Infect. 2, 1051-1060. doi: 10.1016/s1286-4579(00)01259-4

Maeda, T., Garcia-Contreras, R., Pu, M., Sheng, L., Garcia, L. R., Tomas, M., et al. (2012). Quorum quenching quandary: resistance to antivirulence compounds. ISME J. 6, 493-501. doi: 10.1038/ismej.2011.122

Malhotra, S., Limoli, D. H., English, A. E., Parsek, M. R., and Wozniak, D. J. (2018). Mixed communities of mucoid and nonmucoid Pseudomonas aeruginosa exhibit enhanced resistance to host antimicrobials. mBio 9:e275-18. doi: 10 . 1128/mBio.00275-18

Mcmanus, A. T., Mason, A. D., Mcmanus, W. F., and Pruitt, B. A. (1985). Twentyfive year review of Pseudomonas aeruginosa bacteremia in a burn center. Eur. J. Clin. Microbiol. 4, 219-223. doi: 10.1007/bf02013601

Migiyama, Y., Kaneko, Y., Yanagihara, K., Morohoshi, T., Morinaga, Y., Nakamura, S., et al. (2013). Efficacy of AiiM, an N-acylhomoserine lactonase, against Pseudomonas aeruginosa in a mouse model of acute pneumonia. Antimicrob. Agents Chemother. 57, 3653-3658. doi: 10.1128/AAC.00456-13

Mion, S., Remy, B., Plener, L., Bregeon, F., Chabriere, E., and Daude, D. (2019). Quorum quenching lactonase strengthens bacteriophage and antibiotic arsenal against Pseudomonas aeruginosa clinical isolates. Front. Microbiol. 10:2049. doi: 10.3389/fmicb.2019.02049

Morales, E., Gonzalez-Valdez, A., Servin-Gonzalez, L., and Soberon-Chavez, G. (2017). Pseudomonas aeruginosa quorum-sensing response in the absence of functional LasR and LasI proteins: the case of strain 148, a virulent dolphin isolate. FEMS Microbiol. Lett. 364:fnx119. doi: 10.1093/femsle/fnx119

Nguyen, P. D. T., Mustapha, N. A., Kadokami, K., Garcia-Contreras, R., Wood, T. K., and Maeda, T. (2018). Quorum sensing between Gram-negative bacteria responsible for methane production in a complex waste sewage sludge consortium. Appl. Microbiol. Biotechnol. 103, 1485-1495. doi: 10.1007/s00253018-9553-9

Nguyen, P. D. T., Mustapha, N. A., Kadokami, K., Garcia-Contreras, R., Wood, T. K., and Maeda, T. (2019). Quorum sensing between Gram-negative bacteria responsible for methane production in a complex waste sewage sludge consortium. Appl. Microbiol. Biotechnol. 103, 1485-1495. doi: 10.1007/s00253018-9553-9 
Ohman, D. E., Cryz, S. J., and Iglewski, B. H. (1980). Isolation and characterization of Pseudomonas aeruginosa PAO mutant that produces altered elastase. J. Bacteriol. 142, 836-842.

Olivares, E., Badel-Berchoux, S., Provot, C., Jaulhac, B., Prevost, G., Bernardi, T., et al. (2016). The biofilm ring test: a rapid method for routine analysis of Pseudomonas aeruginosa biofilm formation kinetics. J. Clin. Microbiol. 54, 657-661. doi: 10.1128/JCM.02938-15

Papaioannou, E., Wahjudi, M., Nadal-Jimenez, P., Koch, G., Setroikromo, R., and Quax, W. J. (2009). Quorum-quenching acylase reduces the virulence of Pseudomonas aeruginosa in a Caenorhabditis elegans infection model. Antimicrob. Agents Chemother. 53, 4891-4897. doi: 10.1128/AAC.00380-09

Pavlovskis, O. R., and Wretlind, B. (1979). Assessment of protease (elastase) as a Pseudomonas aeruginosa virulence factor in experimental mouse burn infection. Infect. Immun. 24, 181-187.

Rumbaugh, K. P., Diggle, S. P., Watters, C. M., Ross-Gillespie, A., Griffin, A. S., and West, S. A. (2009). Quorum sensing and the social evolution of bacterial virulence. Curr. Biol. 19, 341-345. doi: 10.1016/j.cub.2009.01.050

Shaw, P. D., Ping, G., Daly, S. L., Cha, C., Cronan, J. E. Jr., Rinehart, K. L., et al. (1997). Detecting and characterizing N-acyl-homoserine lactone signal molecules by thin-layer chromatography. Proc. Natl. Acad. Sci. U.S.A. 94, 6036-6041. doi: 10.1073/pnas.94.12.6036

Shields, R. K., Clancy, C. J., Pasculle, A. W., Press, E. G., Haidar, G., Hao, B., et al. (2018). Verification of ceftazidime-avibactam and ceftolozane-tazobactam susceptibility testing methods against carbapenem-resistant Enterobacteriaceae and Pseudomonas aeruginosa. J. Clin. Microbiol. 56:e1093-17. doi: 10.1128/JCM. 01093-17

Shortridge, D., Castanheira, M., Pfaller, M. A., and Flamm, R. K. (2017). Ceftolozane-tazobactam activity against Pseudomonas aeruginosa clinical isolates from U.S. Hospitals: report from the PACTS antimicrobial surveillance program, 2012 to 2015. Antimicrob. Agents Chemother. 61:e465-17. doi: 10.1128/AAC.00465-17

Sio, C. F., Otten, L. G., Cool, R. H., Diggle, S. P., Braun, P. G., Bos, R., et al. (2006). Quorum quenching by an N-acyl-homoserine lactone acylase from Pseudomonas aeruginosa PAO1. Infect. Immun. 74, 1673-1682. doi: 10.1128/ iai.74.3.1673-1682.2006

Soto-Aceves, M. P., Cocotl-Yanez, M., Merino, E., Castillo-Juarez, I., Cortes-Lopez, H., Gonzalez-Pedrajo, B., et al. (2019). Inactivation of the quorum-sensing transcriptional regulators LasR or RhlR does not suppress the expression of virulence factors and the virulence of Pseudomonas aeruginosa PAO1. Microbiology 165, 425-432. doi: 10.1099/mic.0.000778
Tredget, E. E., Shankowsky, H. A., Joffe, A. M., Inkson, T. I., Volpel, K., Paranchych, W., et al. (1992). Epidemiology of infections with Pseudomonas aeruginosa in burn patients: the role of hydrotherapy. Clin. Infect. Dis 15, 941-949. doi: $10.1093 /$ clind/15.6.941

Turner, K. H., Everett, J., Trivedi, U., Rumbaugh, K. P., and Whiteley, M. (2014). Requirements for Pseudomonas aeruginosa acute burn and chronic surgical wound infection. PLoS Genet. 10:e1004518. doi: 10.1371/journal.pgen. 1004518

Utari, P. D., Setroikromo, R., Melgert, B. N., and Quax, W. J. (2018). PvdQ quorum quenching acylase attenuates Pseudomonas aeruginosa virulence in a mouse model of pulmonary infection. Front. Cell Infect. Microbiol. 8:119. doi: $10.3389 /$ fcimb. 2018.00119

Van Delden, C., and Iglewski, B. H. (1998). Cell-to-cell signaling and Pseudomonas aeruginosa infections. Emerg. Infect. Dis 4, 551-560.

Wang, W. Z., Morohoshi, T., Ikenoya, M., Someya, N., and Ikeda, T. (2010). AiiM, a novel class of $\mathrm{N}$-acylhomoserine lactonase from the leaf-associated bacterium Microbacterium testaceum. Appl. Environ. Microbiol. 76, 2524-2530. doi: 10.1128/AEM.02738-09

WHO, (2017). WHO Publishes List of Bacteria for Which New Antibiotics are Urgently Needed. Available at: https://www.who.int/en/news-room/detail/ 27-02-2017-who-publishes-list-of-bacteria-for-which-new-antibiotics-areurgently-needed (accessed February 2, 2018).

Zhang, Y., Sass, A., Van Acker, H., Wille, J., Verhasselt, B., Van Nieuwerburgh, F., et al. (2018). Coumarin reduces virulence and biofilm formation in Pseudomonas aeruginosa by affecting quorum sensing, type III secretion and C-di-GMP levels. Front. Microbiol. 9:1952. doi: 10.3389/fmicb.2018.01952

Conflict of Interest: The authors declare that the research was conducted in the absence of any commercial or financial relationships that could be construed as a potential conflict of interest.

Copyright (c) 2019 López-Jácome, Garza-Ramos, Hernández-Durán, FrancoCendejas, Loarca, Romero-Martínez, Nguyen, Maeda, González-Pedrajo, DíazGuerrero, Sánchez-Reyes, Díaz-Ramírez and García-Contreras. This is an openaccess article distributed under the terms of the Creative Commons Attribution License (CC BY). The use, distribution or reproduction in other forums is permitted, provided the original author(s) and the copyright owner(s) are credited and that the original publication in this journal is cited, in accordance with accepted academic practice. No use, distribution or reproduction is permitted which does not comply with these terms. 